\title{
CIENCIAMATRIA
}

Revista Interdisciplinaria de Humanidades, Educación, Ciencia y Tecnología

Año VII. Vol. VII. N¹2. Enero - Junio. 2021

Hecho el depósito de ley: pp201602FA4721

ISSN-L: 2542-3029; ISSN: 2610-802X

Universidad Nacional Experimental Francisco de Miranda (UNEFM). Santa Ana de Coro. Venezuela

Francisco Agustín Zea-Izquierdo; Juan Edmundo Álvarez-Gavilanes;

Gonzalo Iván Andrade-Mena

DOI $10.35381 / \mathrm{cm} . v 7 i 12.444$

\section{Estudio de mercado del sector automotriz como herramienta para toma de decisiones empresariales}

\section{Market study of the automotive sector as a tool for business decision making}

Francisco Agustín Zea-Izquierdo francisco.zea.60@est.ucacue.edu.ec Universidad Católica de Cuenca, Cuenca

Ecuador

https://orcid.org/0000-0002-7348-3918

Juan Edmundo Álvarez-Gavilanes

juan.alvarezg@ucacue.edu.ec

Universidad Católica de Cuenca, Cuenca

Ecuador

https://orcid.org/0000-0003-0978-3235

Gonzalo Iván Andrade-Mena

gonzaloandrade@ucacue.edu.ec

Universidad Católica de Cuenca, Cuenca

Ecuador

https://orcid.org/0000-0003-2777-4260

Recibido: 01 de octubre de 2020

Aprobado: 15 de diciembre de 2020 


\title{
RESUMEN
}

El conocer un mercado es de mucha importancia sobre todo cuando un inversionista quiere incursionar en él, el objetivo de esta investigación es analizar el mercado de vehículos a diésel en la provincia del Azuay como herramienta de decisiones empresariales para conocer la factibilidad de implementar una empresa distribuidora de repuestos en la 'provincia. La metodología es de tipo descriptiva lo cual permitió conocer a los competidores. El $46 \%$ de los encuestados compran los repuestos para sus vehículos a diésel en la ciudad de Cuenca, además de ser esta la capital provincial. El canal de distribución para estos productos es: importador-distribuidor-cliente final. La empresa proveedora de respuestas deberá estar ubicada en la ciudad de Cuenca de acuerdo a las encuestas realizadas.

Descriptores: Empresa; toma de decisiones; contabilidad; guía de estudios. (Palabras tomadas del Tesauro UNESCO).

\begin{abstract}
Knowing a market is very important especially when an investor wants to enter it, the objective of this research is to analyze the market for diesel vehicles in the province of Azuay as a business decision tool to know the feasibility of implementing a distribution company of spare parts in the 'province. The methodology is descriptive, which allowed us to know the competitors. $46 \%$ of those surveyed buy spare parts for their diesel vehicles in the city of Cuenca, in addition to being the provincial capital. The distribution channel for these products is: importer-distributor-end customer. The answer provider company must be located in the city of Cuenca according to the surveys carried out.
\end{abstract}

Descriptors: Enterprise; decision making; accounting; curriculum guides. (Words taken from the UNESCO Thesaurus). 


\section{INTRODUCCIÓN}

El sector automotriz en el Ecuador en las últimas dos décadas ha tenido mucha importancia para la economía estatal, por el crecimiento sostenido que ha tenido y la cantidad de trabajos y beneficios económicos que genera, tanto para los empresarios que invierten en este sector, como para el país por los aranceles e impuestos que se generan con la actividad (Carrillo, 2009). La Asociación de empresas automotrices del Ecuador AEADE (2018) nos dice que en el sector automotriz. "Desde el año 2016 hasta 2018 se afiliaron 3791 nuevos trabajadores (...). Lo que representa un crecimiento del 5.9\%” (pág. 40). Y este desarrollo sostenido del sector ha llevado al incremento significativo de los recursos fiscales en el año 2018. "738 millones más que en el 2016" (Asociación de Empresas Automotrices Del Ecuador AEADE, 2018, p. 40).

En el Ecuador en los años 2013-2018, el sector automotriz ha tenido un crecimiento promedio anual del 7\%. En el 2018 se matricularon un total de 2403651 vehículos lo que representa el 7.4\% más de lo registrado en el año 2017 que eran 2237264 vehículos; la provincia del Azuay se sitúa como la tercera provincia en número de vehículos por cada 1000 habitantes (181) luego de Tungurahua y Santo domingo de los Tsáchilas según los datos publicados por datos del anuario de transporte 2018 del INEC (Instituto Nacional de Estadística y Censos INEC, 2019). El mercado de vehículos ha ido en constante crecimiento, por la facilidad de crédito que se tiene en la actualidad para acceder a las compras de estos bienes y por la variedad de precios y de tipos de vehículos que existen en el país además también por la eliminación de algunas restricciones que limitaban la importación de vehículos hacia el Ecuador.

Todo lo antes mencionado nos da una idea de la importancia del sector automotriz para las plazas de empleo dentro del país y dentro de la provincia del Azuay. El Instituto Nacional de Estadística y Censos INEC (2012), refiere que la mayoría de establecimientos del sector automotriz se encuentran en Guayas, Pichincha y Azuay, con el $27 \%$, el $17 \%$ y el $8.1 \%$ respectivamente. Siendo la provincia del Azuay la tercera en 


\section{CIENCIAMATRIA \\ Revista Interdisciplinaria de Humanidades, Educación, Ciencia y Tecnología \\ Año VII. Vol. VII. N¹2. Enero - Junio. 2021 \\ Hecho el depósito de ley: pp201602FA4721 \\ ISSN-L: 2542-3029; ISSN: 2610-802X \\ Universidad Nacional Experimental Francisco de Miranda (UNEFM). Santa Ana de Coro. Venezuela}

Francisco Agustín Zea-Izquierdo; Juan Edmundo Álvarez-Gavilanes;

Gonzalo Iván Andrade-Mena

importancia para este sector, para este estudio es importante clasificar al número de vehículos existentes en el país por tipo de combustible que utilizan ya sea: diésel, gasolina, híbrido, eléctrico, gas licuado de petróleo y otros.

En el país el porcentaje de vehículos diésel es del 12.9\%, lo que representa 311009 vehículos que utilizan este tipo de combustible. El sector automotriz en la provincia del Azuay ha tenido un incremento promedio anual del 9.4\% en los años (2013-2018), lo que nos muestra que el crecimiento en esta provincia está por encima del promedio nacional anual, lo que se traduce en 154697 vehículos que existen haciéndolo un mercado muy atractivo para los empresarios del sector automotriz (Instituto Nacional de Estadísticas y Censos INEC, 2019).

En el sector automotriz por tipo de combustible diésel en la provincia del Azuay; el número de vehículos representa el 13.05\% en la provincia lo que nos indica un número de 20202 vehículos lo que lo hace un importante de mercado objetivo (Instituto Nacional de Estadística y Censos INEC, 2018). Pese a que el sector automotriz ha sido uno de los más afectados del país en la crisis sanitaria covid-19 "hay segmentos con buenos resultados. Es el caso de los camiones, cuyas ventas han crecido por cuatro meses consecutivos. Solo en septiembre de 2020, las ventas se elevaron el $11 \%$ en comparación con el mismo mes de 2019" (Coba, 2020).

Los vehículos de tipo de combustible diésel transitan todos los días dentro y fuera de la ciudad y son utilizados en mayor frecuencia para transporte de pasajeros, carga, trabajo pesado, sufren por lo tanto mayor desgaste de sus partes y piezas; y la carencia de establecimientos comerciales dedicados exclusivamente a la distribución de repuestos para vehículos a diésel en la provincia del Azuay nos impone la necesidad de tener una idea clara y concreta del parque automotor nacional y provincial por el tipo de combustible que utilizan los vehículos.

El objetivo de esta investigación es realizar un estudio de mercado para analizar el mercado potencial del sector automotriz diésel en la provincia del Azuay para de esta 


\section{CIENCIAMATRIA \\ Revista Interdisciplinaria de Humanidades, Educación, Ciencia y Tecnología \\ Año VII. Vol. VII. N¹2. Enero - Junio. 2021 \\ Hecho el depósito de ley: pp201602FA4721 \\ ISSN-L: 2542-3029; ISSN: 2610-802X \\ Universidad Nacional Experimental Francisco de Miranda (UNEFM). Santa Ana de Coro. Venezuela}

Francisco Agustín Zea-Izquierdo; Juan Edmundo Álvarez-Gavilanes;

Gonzalo Iván Andrade-Mena

manera posteriormente conocer la factibilidad de la implementación de una empresa distribuidora de repuestos diésel que esté orientada a dar soluciones de partes y piezas para vehículos que utilicen este tipo de combustible, desde la percepción del posible consumidor, aplicarlo como herramienta de decisión empresarial, el cual ayude también al progreso de la comunidad al dar empleo, contribuya de cierta forma a la economía local, y evite la pérdida de tiempo de los posibles clientes al no conseguir un repuesto para de este tipo.

\section{Referencial Teórico}

\section{El estudio de mercado como herramienta de toma de decisiones empresariales para analizar la factibilidad de un proyecto.}

Al realizar un estudio de mercado el empresario, o la parte interesada lo que busca es reducir el riesgo al momento de tomar una decisión, lo cual es de mucha importancia ya que nos permite obtener información clara y precisa sobre el mercado que se va a estudiar y disminuye el riesgo de la inversión (Ferré-Trenzano \& Ferré-Nadal, 1997). Al respecto, el Instituto Latinoamericano de Planificación y Economía Social ILPES (2006) sostiene que el estudio de mercado consiste en "probar que existe un número suficiente de individuos, que, dadas ciertas condiciones, presentan una demanda que justifica la puesta en marcha de un proyecto en cierto periodo". (p. 72)

Siempre es importante realizar un estudio de mercado antes de tomar una decisión empresarial, ya que se tendrá conocimiento previo de la demanda insatisfecha a la cual se puede satisfacer ofreciendo el producto, esto permite determinar el mercado en el que se va a incursionar, los precios y la competencia. Es así que mediante este estudio de mercado se busca determinar la factibilidad de la implementación de una empresa dedicada a la comercialización de partes y piezas para vehículos a diésel en la provincia del Azuay desde la percepción del consumidor. 


\section{CIENCIAMATRIA \\ Revista Interdisciplinaria de Humanidades, Educación, Ciencia y Tecnología \\ Año VII. Vol. VII. N¹2. Enero - Junio. 2021 \\ Hecho el depósito de ley: pp201602FA4721 \\ ISSN-L: 2542-3029; ISSN: 2610-802X \\ Universidad Nacional Experimental Francisco de Miranda (UNEFM). Santa Ana de Coro. Venezuela}

Francisco Agustín Zea-Izquierdo; Juan Edmundo Álvarez-Gavilanes;

Gonzalo Iván Andrade-Mena

Para realizar un estudio de mercado de una manera correcta se debe considerar 4 mercados a analizar dentro del sector al que se refiere el estudio: el mercado proveedor, el mercado competidor o la oferta, el mercado distribuidor y el mercado consumidor o la demanda, cada elemento a estudiar aporta información muy útil para evaluar el proyecto (Sapag-Chain, 2007).

De igual manera (Sellers-Rubio \& Casado-Díaz, 2013), detallan los pasos a seguir para un estudio de mercado, para lo cual primero se debe saber el mercado al que el estudio se va a dirigir, es decir un conjunto de consumidores que tengan la necesidad similar, la capacidad y que además estén dispuestos a comprar los productos que la empresa ofrezca para resolver su problema; como segundo punto se debe calcular la demanda de los productos que se van a ofrecer y tratar de predecirlas a futuro para la duración estimada del proyecto es decir un análisis cuantitativo; posteriormente como tercer punto se debe analizar el mercado de forma cualitativa, es decir conocer el porqué, como, cuando y donde consume el producto el cliente de acuerdo con su necesidad, analizar las fortalezas así como las debilidades que existen en el sector y finalmente como punto cuatro en el análisis se debe dividir al mercado en segmentos de características similares que son importantes para el consumo del bien o servicio, y que además esta segmentación nos dirá cuál es el mercado objetivo al que el proyecto se va a dirigir y permitirá encontrar las estrategias para enfocarse en dicho mercado objetivo (Álvarez, et al., 2020).

El realizar un estudio de mercado es importante para el éxito del proyecto que se desea alcanzar ya que ofrece información pasada, presente y futura del sector al que está dirigida la idea de negocio y permite conocer la factibilidad de la idea indicando si debe continuar con un riesgo menor o cambiar de plan. Para realizar el estudio se debe establecer los objetivos, determinar qué tipo de información necesitamos, recolectarla y analizarla para elaborar un informe con los resultados del análisis de mercado. En la presentación del estudio se debe analizar la demanda, la oferta y el análisis de 


\section{CIENCIAMATRIA \\ Revista Interdisciplinaria de Humanidades, Educación, Ciencia y Tecnología \\ Año VII. Vol. VII. N¹2. Enero - Junio. 2021 \\ Hecho el depósito de ley: pp201602FA4721 \\ ISSN-L: 2542-3029; ISSN: 2610-802X \\ Universidad Nacional Experimental Francisco de Miranda (UNEFM). Santa Ana de Coro. Venezuela}

Francisco Agustín Zea-Izquierdo; Juan Edmundo Álvarez-Gavilanes;

Gonzalo Iván Andrade-Mena

comercialización además de las $4 \mathrm{P}$ del marketing: plaza, precio, promoción y producto (C.E.E.I Galicia, S.A. (BIC Galicia), 2010).

Acerca del estudio de mercado como herramienta para tomar deciones Vélez-Pareja (2003) afirma "Es algo natural en el ser humano. Siempre se están tomando decisiones y muchas veces no nos percatamos de ello" (pág. 1) al realizar el análisis de mercado es una forma de "visualizar la realidad (...) en forma sencilla, sintética y práctica" (VélezPareja, 2003, p. 2).

La obtención de información relevante para planificar y controlar un proyecto para estudiar un mercado, disminuye el riesgo de desacierto en la toma de decisiones, y al obtener esta información de calidad obtenemos mejores resultados con más seguridad de éxito enfocado en las necesidades del mercado (Kinnear \& Taylor, 1991).

\section{Investigación de mercado pasos, características y análisis.}

Cuando se tiene un proyecto que al parecer es realizable y se ejecuta un estudio de mercado que se enfoca en el entendimiento de los fragmentos del mercado que afecten al proyecto, se puede anticipar la respuesta de los consumidores y plantear estrategias comerciales que lleven la idea al éxito del negocio luego del análisis del macro entorno y del micro entorno (García-González \& Bória-Reverter, 2006). Lo primero que tenemos que realizar en el estudio de mercado es definir el tamaño total de la población objeto de nuestro estudio para conocer el beneficio económico que puede generar la implementación del proyecto, tratando de predecir lo que va a pasar a través del estudio de factibilidad.

Según (Griffin \& Ebert, 2005) El mercado objetivo son todas las personas que muestran interés en los mismos productos con las mismas necesidades y deseos; además definen también lo que es un segmento de mercado en el cual se dividen por categorías de acuerdo con variables demográficas y geográficas que son las que utilizaremos para dividir la población objeto de nuestro estudio (Erazo \& Narváez, 2020). 
Al identificar la demanda en su totalidad que está ubicada dentro de una población y que está definida por su alcance, ya sea estatal, provincial o municipal, debemos delimitar las características en común que tiene la población objeto de investigación, precisar la unidad de análisis y la población en un conjunto que concuerde con las especificaciones necesarias para el análisis de su comportamiento como demanda (Luna-González, 2016). El mercado al cual está enfocado este estudio es al mercado de vehículos que utilizan de tipo de combustible diésel en la provincia del Azuay, ya que presenta una demanda insatisfecha debido a que existen pocos locales que comercializan partes y piezas para estos vehículos.

Es necesario para esto conocer el mercado total de vehículos y analizar los datos históricos que existen primero de crecimiento anual de vehículos en el Ecuador y luego de crecimiento anual en la provincia del Azuay, y luego subdividirlos por tipo de combustible que utilizan los automotores para tener una idea clara del mercado de vehículos al cual enfocamos esta investigación.

El sector de los proveedores de partes y piezas automotrices es amplio y variado lo que permite que este tenga un desarrollo constante en el ámbito comercial, a medida que la industria automotriz ha ido creciendo en el planeta, este sector ha necesitado más mano de obra a nivel mundial y se estima que avance más rápido incluso que el propio sector de la fabricación de automotores (Organización internacional del trabajo, 2005). De igual forma (Ortega-Bardellini, 2005) refiere que el sector automotriz en el mundo es uno de los sectores más competitivos, por lo que es importante para el estudio de mercado automotriz ecuatoriano identificar las fortalezas y debilidades del entorno nacional para conocer la estructura del mercado y del lugar en el que se desenvuelve, establecer el nivel de competitividad y el potencial a futuro que puede llegar a tener.

Para poder comprender el mercado ecuatoriano de vehículos y poder establecer nuestro mercado objetivo a continuación, en la tabla 1, observamos el crecimiento nacional 


\section{CIENCIAMATRIA}

Revista Interdisciplinaria de Humanidades, Educación, Ciencia y Tecnología

Año VII. Vol. VII. N¹2. Enero - Junio. 2021

Hecho el depósito de ley: pp201602FA4721

ISSN-L: 2542-3029; ISSN: 2610-802X

Universidad Nacional Experimental Francisco de Miranda (UNEFM). Santa Ana de Coro. Venezuela

Francisco Agustín Zea-Izquierdo; Juan Edmundo Álvarez-Gavilanes;

Gonzalo Iván Andrade-Mena

histórico anual que han tenido los vehículos en el Ecuador en los últimos años en número de vehículos y en porcentaje.

\section{Tabla1.}

Número de vehículos matriculados por años en el Ecuador.

\begin{tabular}{lll}
\hline Año & $\begin{array}{l}\text { Número } \\
\text { vehículos en } \\
\text { el país }\end{array}$ & $\begin{array}{l}\text { Porcentaje } \\
\text { crecimiento }\end{array}$ \\
\hline $\mathbf{2 0 1 3}$ & 1719957 & \\
\hline $\mathbf{2 0 1 4}$ & 1752712 & $1,9 \%$ \\
\hline $\mathbf{2 0 1 5}$ & 1925368 & $9,8 \%$ \\
\hline $\mathbf{2 0 1 6}$ & 2056213 & $6,8 \%$ \\
\hline $\mathbf{2 0 1 7}$ & 2237264 & $8,8 \%$ \\
\hline $\mathbf{2 0 1 8}$ & 2403651 & $7,4 \%$ \\
\hline
\end{tabular}

Fuente: Instituto Nacional de Estadística y Censos INEC (2019).

Del total de vehículos existentes en el año 2018 en el Ecuador para este estudio se subdivide el número de vehículos en el Ecuador según tipo de combustible que utilizan según datos del instituto nacional de estadística y censos como podemos observar en la tabla 2. 


\section{Tabla 2.}

Vehículos motorizados matriculados según uso de vehículo, año 2018 por clase y tipo de combustible.

\begin{tabular}{|c|c|c|c|c|c|c|}
\hline \multirow[t]{2}{*}{ Clase } & \multirow{2}{*}{$\begin{array}{l}\text { Tipo de } \\
\text { combustible }\end{array}$} & \multirow[t]{2}{*}{ Total } & \multicolumn{4}{|l|}{ Uso } \\
\hline & & & Estado & Alquiler & Particular & Otros \\
\hline \multirow[t]{6}{*}{ Total } & Diésel & 311.009 & 14.493 & 94.827 & 201.559 & 130 \\
\hline & Gasolina & 2.082 .329 & 20.718 & 83.309 & 1.977 .509 & 793 \\
\hline & Híbrido & 9.655 & 48 & 2 & 9.600 & 5 \\
\hline & Eléctrico & 276 & 7 & 35 & 234 & - \\
\hline & $\begin{array}{l}\text { Gas licuado de } \\
\text { petróleo }\end{array}$ & 159 & 3 & 6 & 150 & - \\
\hline & Otro & 223 & 6 & 6 & 211 & - \\
\hline
\end{tabular}

Fuente: Instituto Nacional de Estadística y Censos INEC (2018).

Para poder segmentar el mercado es importante analizar algunas variables, la primera en función de las características que describen a los consumidores siendo estas: Geográficas que es el lugar donde se encuentra el segmento de mercado, demográficas que divide al grupo en edad, ingresos, género y psicográficas que divide al grupo estilo de vida y personalidad. Como segunda variable se debe analizar si dichos segmentos presentan diferentes necesidades ante los productos que se van a ofrecer (Kotler \& Keller, 2006).

Ahora en la tabla 3 del estudio analizaremos el crecimiento total de vehículos en la provincia del Azuay que es el segmento demográfico al cual enfocamos este estudio de mercado. 


\section{CIENCIAMATRIA}

Revista Interdisciplinaria de Humanidades, Educación, Ciencia y Tecnología

Año VII. Vol. VII. N¹2. Enero - Junio. 2021

Hecho el depósito de ley: pp201602FA4721

ISSN-L: 2542-3029; ISSN: 2610-802X

Universidad Nacional Experimental Francisco de Miranda (UNEFM). Santa Ana de Coro. Venezuela

Francisco Agustín Zea-Izquierdo; Juan Edmundo Álvarez-Gavilanes;

Gonzalo Iván Andrade-Mena

\section{Tabla 3.}

Número de vehículos matriculados por años en la provincia del Azuay.

\begin{tabular}{lll}
\hline Año & Número vehículos en Azuay & $\begin{array}{l}\text { Porcentaje } \\
\text { crecimiento } \\
\text { en Azuay }\end{array}$ \\
\hline $\mathbf{2 0 1 3}$ & 99.972 & \\
\hline $\mathbf{2 0 1 4}$ & 105.178 & $5,2 \%$ \\
\hline $\mathbf{2 0 1 5}$ & 124.069 & $18 \%$ \\
\hline $\mathbf{2 0 1 6}$ & 120.331 & $-3,1 \%$ \\
\hline $\mathbf{2 0 1 7}$ & 141848 & $17,8 \%$ \\
\hline $\mathbf{2 0 1 8}$ & 154.697 & $9,1 \%$ \\
\hline
\end{tabular}

Fuente: Instituto Nacional de Estadística y Censos INEC (2018).

Una vez conocido el número de vehículos existentes en la provincia del Azuay de manera igual subdividimos el número total de vehículos existentes por tipo de combustible que utilizan para obtener el segmento de mercado al cual vamos a dirigir esta investigación como podemos observar en la tabla 4. 


\section{Tabla 4.}

Vehículos motorizados matriculados según uso de vehículo, año 2018 por clase y tipo de combustible.

\begin{tabular}{|c|c|c|c|c|c|c|}
\hline Provincia & $\begin{array}{l}\text { Tipo } \\
\text { combustible }\end{array}$ & Total & Estado & Alquiler & Particular & Otros \\
\hline \multirow[t]{6}{*}{ Azuay } & Diésel & 20.202 & 885 & 5.089 & 14.226 & 2 \\
\hline & Gasolina & 133.934 & 1.050 & 5.356 & 127.512 & 16 \\
\hline & Híbrido & 515 & 3 & - & 512 & - \\
\hline & Eléctrico & 19 & 2 & - & 17 & - \\
\hline & $\begin{array}{l}\text { Gas licuado } \\
\text { de petróleo }\end{array}$ & 14 & - & 1 & 13 & - \\
\hline & Otro & 13 & 1 & 1 & 11 & - \\
\hline
\end{tabular}

Fuente: Instituto Nacional de Estadística y Censos INEC (2018).

El mercado objetivo de este estudio es de 20202 vehículos que utilizan de tipo de combustible diésel; este mercado de la provincia del Azuay es de mucha importancia ya que representa el $6.5 \%$ del total del mercado nacional siendo un número importante y atractivo para analizar su factibilidad.

\section{Estrategias de mercados para conseguir objetivos planteados en el estudio}

Una vez definido el tamaño de la población lo siguiente a realizar es el análisis de proveedores, competidores, distribuidores y consumidores.

El mercado proveedor consta de tres aspectos importantes para su estudio que son: precios de insumos que determinan la cantidad de la inversión; disponibilidad en la existencia y calidad de los productos.

El mercado competidor o análisis de la oferta es aquel que se enfoca en conocer el funcionamiento de empresas de características iguales a las del proyecto que se está 
estudiando que ofrezcan bienes de cualidades similares y ayuda a implementar estrategias comerciales competitivas.

El mercado distribuidor es la cadena de distribución de los productos hasta llegar al consumidor final.

El mercado consumidor o análisis de la demanda es el más importante para el proyecto en el cual existen componentes: racionales como comparación de precios, formas de pago, calidad, prestigio empresarial y componentes emocionales como moda, marca y confianza sobre las características que no se pueden medir como servicio post-venta y existencia de repuestos para la adquisición del bien y nos permite conocer cuáles son las características de necesidades similares que tienen los clientes que buscan satisfacer una necesidad (Sapag-Chain, 2007).

En el caso de las partes y piezas para vehículos los proveedores son todas aquellas concesionarias de vehículos y los importadores de partes y piezas de vehículos a diésel, la mayoría ubicadas en la provincia del Guayas y Pichincha. Los precios de las partes y piezas de los automotores a diésel en las concesionarias son un poco más altos que del que ofrecen los importadores ya que estos productos son genuinos y de la más alta calidad.

En cambio, los precios de los importadores son más económicos ya que estos son repuestos genéricos, y la calidad de los productos se define mediante el lugar de procedencia de los productos. Luego de realizado el análisis de proveedores, realizamos el análisis de la competencia para nuestro estudio. En el Azuay existen muy pocos locales dedicados a la venta de partes y piezas para vehículos a diésel, lo que hace que sea un mercado atractivo para la entrada de nuevas empresas que se dediquen a esta actividad. Existen 5 locales en la provincia del Azuay que son los más importantes, y que son competencia directa entre ellos están Eco diésel, Más diésel, Diésel Motors, Repuestos diésel y Austro diésel. 


\section{CIENCIAMATRIA \\ Revista Interdisciplinaria de Humanidades, Educación, Ciencia y Tecnología \\ Año VII. Vol. VII. N¹2. Enero - Junio. 2021 \\ Hecho el depósito de ley: pp201602FA4721 \\ ISSN-L: 2542-3029; ISSN: 2610-802X \\ Universidad Nacional Experimental Francisco de Miranda (UNEFM). Santa Ana de Coro. Venezuela}

Francisco Agustín Zea-Izquierdo; Juan Edmundo Álvarez-Gavilanes;

Gonzalo Iván Andrade-Mena

La competencia indirecta sería el mercado de repuestos usados o de segunda mano y las concesionarias de los vehículos que también distribuyen repuestos a estas empresas, y a los clientes finales. Esto nos muestra que es un mercado con poca oferta y mucha demanda debido al gran número de vehículos diésel existentes en la provincia del Azuay. Ahora analizaremos el mercado consumidor; al tener definido ya el mercado potencial se debe definir porque los clientes prefieren comprar en un lugar determinado, analizando sus tendencias de compra y sus gustos en cuanto a la elección del lugar en el que realizan la compra, ya sea por factores racionales como precio, calidad, etc. o subjetivos como pueden ser inventario de repuestos, servicio post-venta, (Zhingre, et a., 2020).

El marketing es el grupo de técnicas que con los estudios de mercados pretenden tener el mayor beneficio de un producto y conocer a qué público le interesa, satisfaciendo al cliente potencial y permitiendo diseñar el producto, fijando los precios, los canales de distribución y las técnicas de comunicación esto es conocido como el marketing mis que diseña las estrategias para alcanzar los objetivos, estas herramientas son conocidas como las 4 P: precio, plaza, promoción y producto (Olea-Suaréz \& Pacheco-Garcia, 2012).

Para continuar con el estudio de mercado, se necesita analizar las $4 \mathrm{P}$ del marketing o Marketing mix para tener una idea más clara del mercado al cual dirigimos este estudio. Dvoskin (2004) afirma. "No podemos analizar una P sin tener en cuenta las otras tres, porque en la práctica funcionan como unidad indisoluble" (p. 29). Además, (PedrazaRendon, 2014), al respecto dice que el producto es la utilidad del bien que cumple con las expectativas del cliente; precio es lo que el cliente recibe a cambio de su dinero, ya sea un bien o un servicio de calidad; promoción es la forma de ofrecer efectivamente los productos a los clientes para que estos eligan nuestro proyecto y plaza los canales de distribución que se utilizan para llegar con el producto al consumidor. El sector en el que se ofrece el bien está influenciado por organizaciones que venden productos de 


\section{CIENCIAMATRIA \\ Revista Interdisciplinaria de Humanidades, Educación, Ciencia y Tecnología \\ Año VII. Vol. VII. N¹2. Enero - Junio. 2021 \\ Hecho el depósito de ley: pp201602FA4721 \\ ISSN-L: 2542-3029; ISSN: 2610-802X \\ Universidad Nacional Experimental Francisco de Miranda (UNEFM). Santa Ana de Coro. Venezuela}

Francisco Agustín Zea-Izquierdo; Juan Edmundo Álvarez-Gavilanes;

Gonzalo Iván Andrade-Mena

características similares y atienden al mismo mercado objetivo esto afecta directamente la operación del negocio objeto de estudio.

El análisis de producto no solo es el ofrecimiento del bien para satisfacer la necesidad, también se debe sumar una propuesta de valor, la aceptabilidad de este para ofrecer la solución al cliente (Sutil-Martín, 2013). El análisis de precio, para el análisis de esta variable se consideran dos aspectos importantes: el costo del producto que se va a ofrecer, y los precios que el mercado está dispuesto a pagar, este debe ser un precio atractivo a que los clientes adquieran el producto (Parmerlee, 1999).

El análisis de promoción es la comunicación de la empresa con el cliente, a más del bien de calidad que se ofrece, a un precio atractivo para el mercado, a través de un canal de distribución correctamente estructurado, se debe dar a conocer al cliente el producto y lo que diferencia de la competencia, para alcanzar buena reputación que está permita estimular la compra del bien por acciones promocionales (Talaya, y otros, 2008). El análisis de plaza o canal de distribución del producto define las funciones y los niveles de intermediarios que el bien recorre hasta llegar al cliente final (Dvoskin, 2004).

Para concluir el estudio de mercado se debe elaborar un informe claro y preciso de la información recolectada en el análisis de mercado; siendo este informe una parte fundamental del proceso metodológico de la investigación ya que este conforma el medio de contacto entre los resultados obtenidos y la persona u organización interesada en el proyecto para el cual se realiza el estudio de mercado (Fernández-Nogales, 2004).

\section{MÉTODO}

Para la obtención de información para el estudio de mercado; la investigación es descriptiva no experimental. Para este análisis de mercado se utilizó un muestreo por conveniencia no aleatorio que es un tipo de muestreo no probabilístico a 78 personas propietarias de vehículos a diésel que viven dentro de la provincia del Azuay. 
La herramienta de obtención de información se la creo a través de un formulario de Google (https://docs.google.com.forms/) para que la recolección de datos sea sencilla y rápida debido a la pandemia COVID-19, misma que contenía 6 preguntas las cuales nos brindaron información sobre competencia, cliente, mercado; para la tabulación de datos y gráficos obtenidos en el formulario se utilizó la herramienta informática Microsoft Excel 2016.

\section{RESULTADOS}

Los resultados de la encuesta dirigida a los propietarios de vehículos diésel en la provincia del Azuay nos plantean los siguientes resultados.

\section{Dimensión: Clientes}

Los clientes potenciales consideran en el $96 \%$ que es importante la garantía del producto, el $73 \%$ considera importante el servicio postventa y el $68 \%$ considera importante la calidad del servicio; lo que nos indica los factores más importantes a ser tomados en cuenta. Además, prefieren en el $47 \%$ que el local se encuentre ubicado en la ciudad de Cuenca. El $51 \%$ de los encuestados compra repuestos dos veces al año y el $45 \%$ gasta entre 250 y 500 dólares cada vez que compra repuestos para su vehículo a diésel.

\section{Dimensión: Producto}

El $49 \%$ de los encuestados indica que los repuestos que sufren mayor desgaste o daño son los repuestos de motor seguidos del $11.1 \%$ repuestos de caja de cambios y el $9,9 \%$ repuestos eléctricos lo que nos indica cuales serían los productos de mayor rotación.

Los demás resultados los analizamos en el siguiente paso de este estudio de mercado que es la propuesta. 


\section{CIENCIAMATRIA}

Revista Interdisciplinaria de Humanidades, Educación, Ciencia y Tecnología

Año VII. Vol. VII. N¹2. Enero - Junio. 2021

Hecho el depósito de ley: pp201602FA4721

ISSN-L: 2542-3029; ISSN: 2610-802X

Universidad Nacional Experimental Francisco de Miranda (UNEFM). Santa Ana de Coro. Venezuela

Francisco Agustín Zea-Izquierdo; Juan Edmundo Álvarez-Gavilanes;

Gonzalo Iván Andrade-Mena

\section{PROPUESTA}

Para estudiar un mercado involucra conocer todos los ámbitos que lo afectan, para que el estudio en cuestión sea real y dé una idea clara del mercado al que la parte interesada quiere entrar, se debe analizar seis pasos que en su esquema nos indica debemos seguir como podemos apreciar en la figura 1.

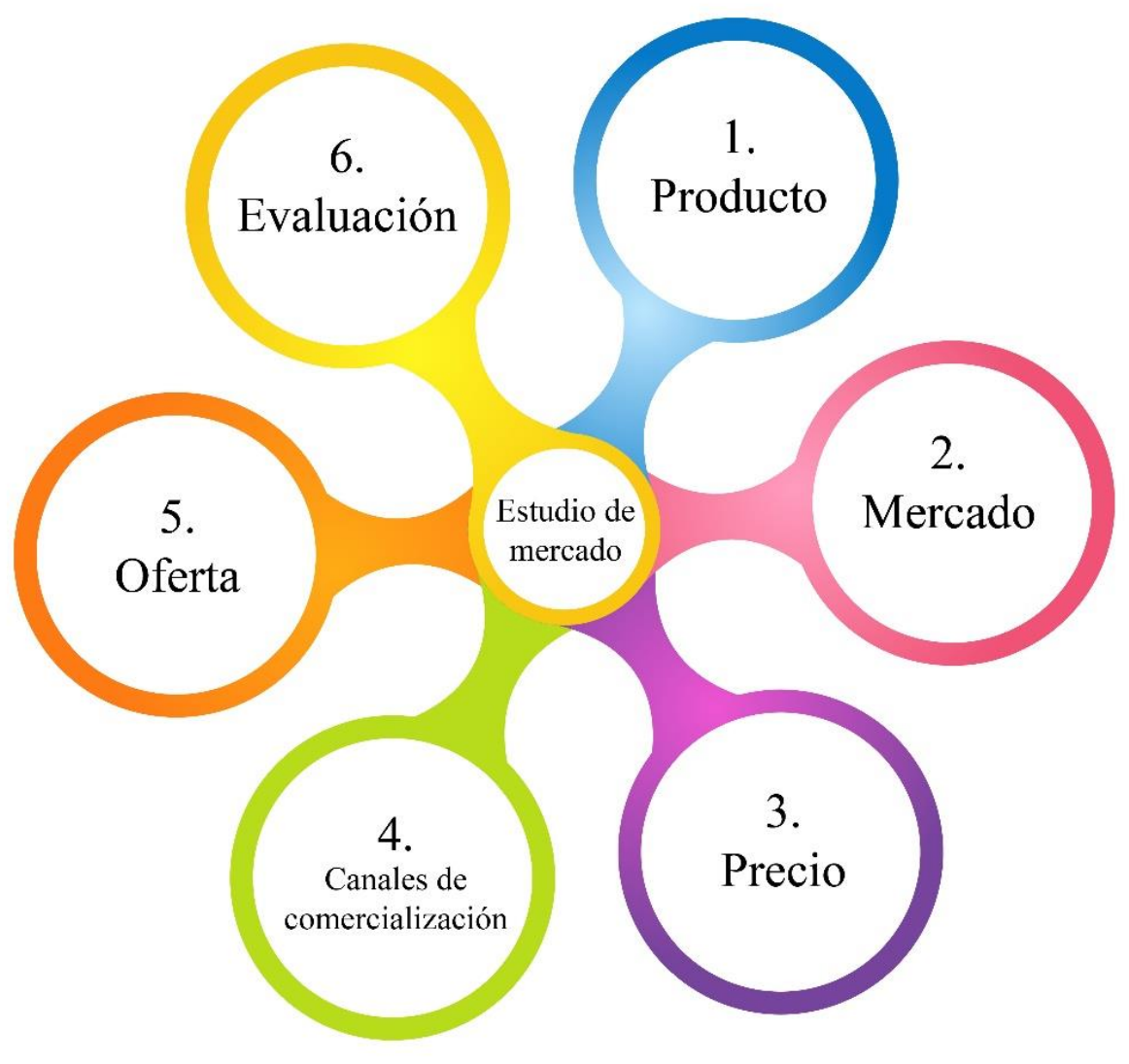

Figura 1. Esquema de la propuesta.

La intención al realizar este estudio de mercado es saber la factibilidad de la implementación de una empresa distribuidora de partes y piezas para vehículos a diésel en la provincia del Azuay. 


\section{CIENCIAMATRIA}

Revista Interdisciplinaria de Humanidades, Educación, Ciencia y Tecnología

Año VII. Vol. VII. N¹2. Enero - Junio. 2021

Hecho el depósito de ley: pp201602FA4721

ISSN-L: 2542-3029; ISSN: 2610-802X

Universidad Nacional Experimental Francisco de Miranda (UNEFM). Santa Ana de Coro. Venezuela

Francisco Agustín Zea-Izquierdo; Juan Edmundo Álvarez-Gavilanes;

Gonzalo Iván Andrade-Mena

\section{Producto}

Los productos que se ofrecen son repuestos para vehículos a diésel; que son partes iguales a las que se encuentran puestas en el vehículo, y están diseñadas para ser sustituidas cuando estas se han deteriorado o han cumplido ya su vida útil, y las podemos clasificar por el lugar en donde estas se ubican como podemos apreciar en la figura 2.

\section{Repuestos de motor}

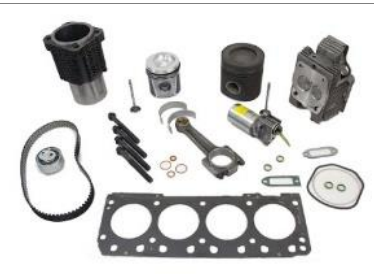

\section{Repuestos caja de cambios}

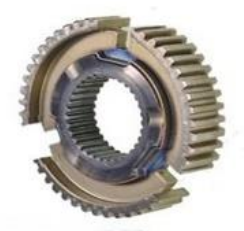

\section{Repuestos eléctricos}

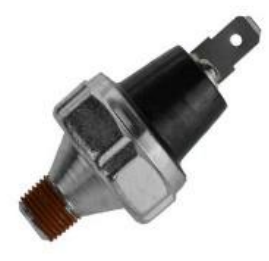

\section{Repuestos de sistema de inyección}

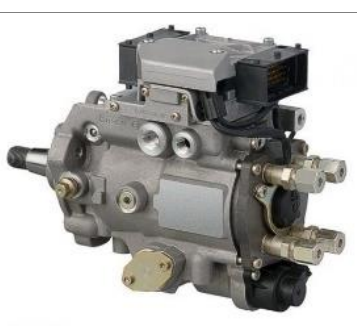




\section{Repuestos de suspensión}

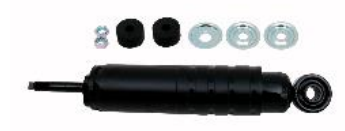

\section{Repuestos de transmisión}

Figura 2. Tipos de repuestos en un vehículo de tipo de combustible diésel. Elaboración propia.

\section{Mercado}

El mercado al que se dirige este estudio es a los propietarios de vehículos a diésel en la provincia del Azuay parra lo cual primero vemos el crecimiento anual de vehículos en la provincia; y luego dividimos en número de vehículos por tipo de combustible que utilizan.

\section{Tabla 5.}

Vehículos matriculados por año en la provincia del Azuay.

\begin{tabular}{ll}
\hline Año & Número de vehiculos \\
\hline 2014 & 105178 \\
\hline 2015 & 124069 \\
\hline 2016 & 120331 \\
\hline 2017 & 141848 \\
\hline 2018 & 154697
\end{tabular}

Fuente: Instituto Nacional de Estadística y Censos INEC (2018). 


\section{CIENCIAMATRIA}

Revista Interdisciplinaria de Humanidades, Educación, Ciencia y Tecnología

Año VII. Vol. VII. N¹2. Enero - Junio. 2021

Hecho el depósito de ley: pp201602FA4721

ISSN-L: 2542-3029; ISSN: 2610-802X

Universidad Nacional Experimental Francisco de Miranda (UNEFM). Santa Ana de Coro. Venezuela

Francisco Agustín Zea-Izquierdo; Juan Edmundo Álvarez-Gavilanes;

Gonzalo Iván Andrade-Mena

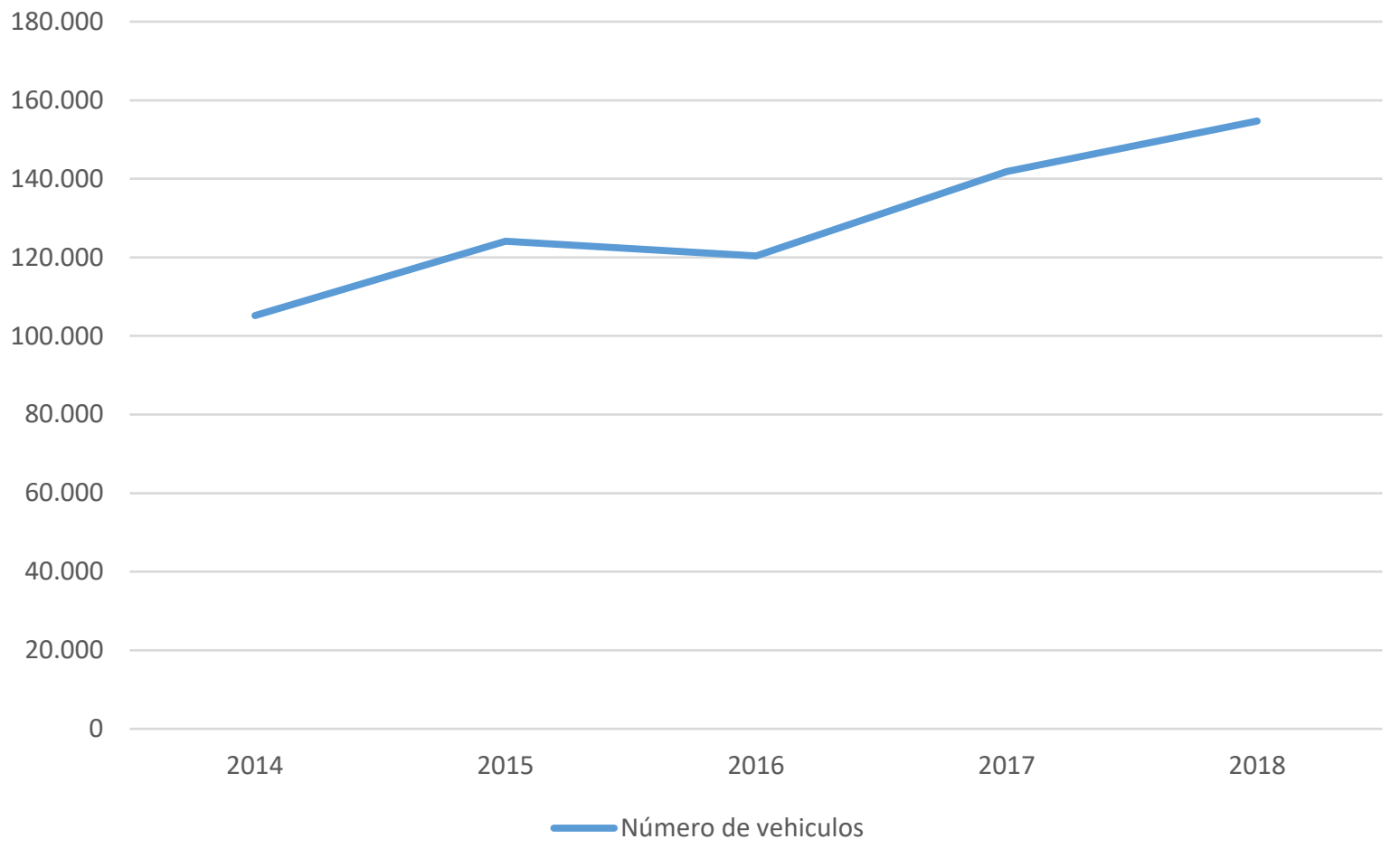

Figura 3. Vehículos matriculados por año en la provincia del Azuay.

Tabla 6.

Número de vehículos por tipo de combustible que utilizan en la provincia del Azuay año 2018.

Tipo de combustible

Diésel

Gasolina

Otros
Número de Vehículos

20202

133934

561

Fuente: Instituto Nacional de Estadística y Censos INEC (2018). 


\section{CIENCIAMATRIA}

Revista Interdisciplinaria de Humanidades, Educación, Ciencia y Tecnología

Año VII. Vol. VII. N¹2. Enero - Junio. 2021

Hecho el depósito de ley: pp201602FA4721

ISSN-L: 2542-3029; ISSN: 2610-802X

Universidad Nacional Experimental Francisco de Miranda (UNEFM). Santa Ana de Coro. Venezuela

Francisco Agustín Zea-Izquierdo; Juan Edmundo Álvarez-Gavilanes;

Gonzalo Iván Andrade-Mena

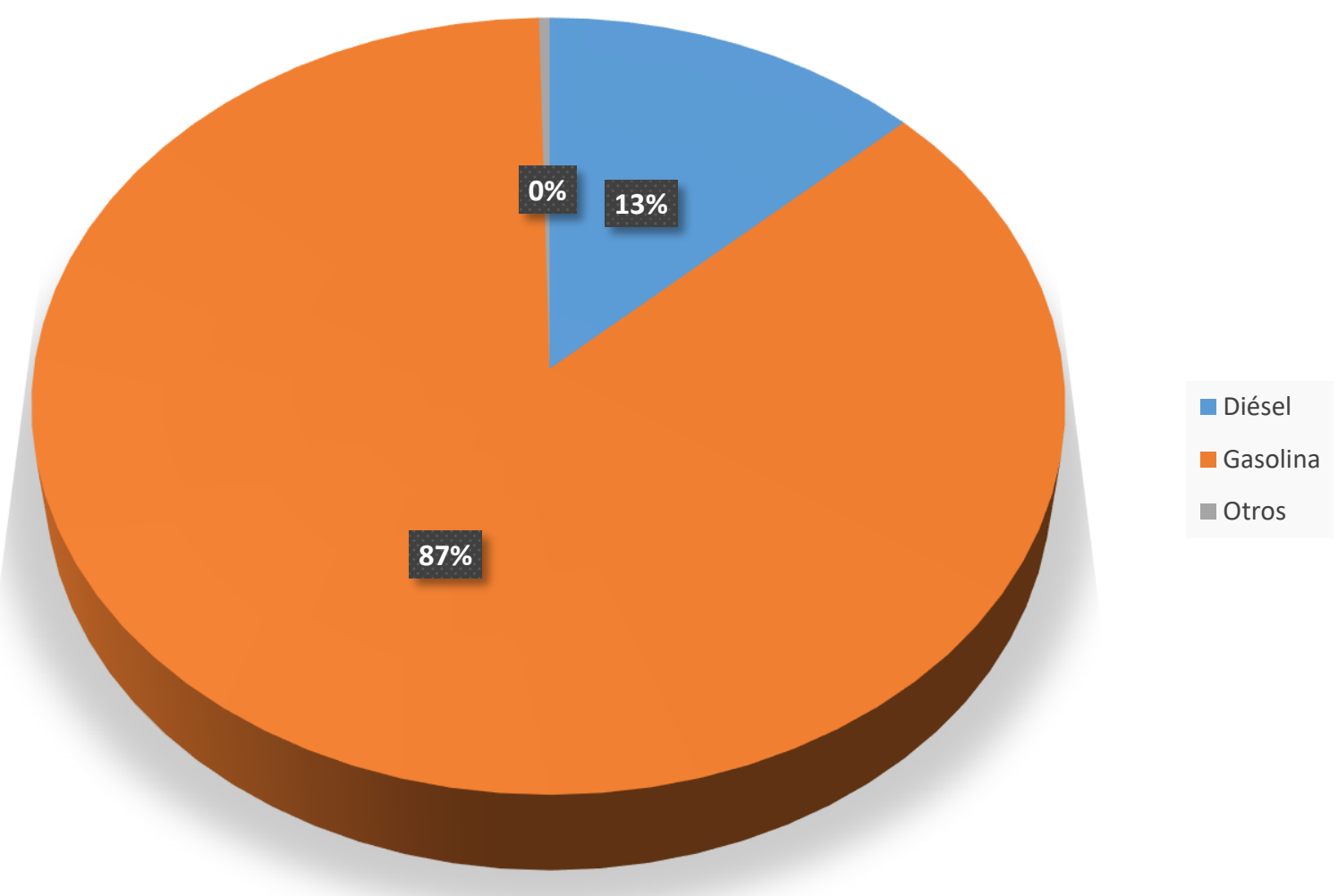

Figura 4. Número de vehículos por tipo de combustible que utilizan en la provincia del Azuay año 2018.

Al observar los gráficos podemos decir que el mercado de vehículos está en constante desarrollo y crecimiento dándonos la idea clara de que es atractivo invertir en él y los vehículos diésel que son nuestro mercado objetivo son el 13\% del total representando así una cuota interesante de mercado del total de vehículos existentes en la provincia del Azuay. 


\section{CIENCIAMATRIA}

Revista Interdisciplinaria de Humanidades, Educación, Ciencia y Tecnología

Año VII. Vol. VII. N¹2. Enero - Junio. 2021

Hecho el depósito de ley: pp201602FA4721 ISSN-L: 2542-3029; ISSN: 2610-802X

Universidad Nacional Experimental Francisco de Miranda (UNEFM). Santa Ana de Coro. Venezuela

Francisco Agustín Zea-Izquierdo; Juan Edmundo Álvarez-Gavilanes;

Gonzalo Iván Andrade-Mena

\section{Precio}

Para definir los precios y las promociones de los productos se fijarán estrategias las cuales veremos a continuación en la siguiente matriz.

\section{Política de precios y promociones}

Estrategia de precios.

Fijar los precios de los productos para

el mercado objetivo.

Estrategia de promociones.

Forma en como dar a conocer el producto a los clientes
- Precios similares a los de la competencia con productos de calidad similar.

- Margen de utilidad razonable para el empresario.

- Precio de costo y transporte de los productos.

- Anunciar productos en redes sociales.

- Ponerse en contacto con dueños de talleres de vehículos a diésel.

- Alianzas estratégicas con empresas que requieran de repuestos para vehículos a diésel.

\section{Canales de Distribución}

El canal de distribución para estos productos es: importador-distribuidor-cliente final.

La empresa deberá estar ubicada en la ciudad de Cuenca de acuerdo a las encuestas realizadas, ya que el $46 \%$ de los encuestados compran los repuestos para sus vehículos a diésel en la ciudad de Cuenca, además de ser esta la capital provincial. 


\section{CIENCIAMATRIA}

Revista Interdisciplinaria de Humanidades, Educación, Ciencia y Tecnología

Año VII. Vol. VII. N¹2. Enero - Junio. 2021

Hecho el depósito de ley: pp201602FA4721

ISSN-L: 2542-3029; ISSN: 2610-802X

Universidad Nacional Experimental Francisco de Miranda (UNEFM). Santa Ana de Coro. Venezuela

Francisco Agustín Zea-Izquierdo; Juan Edmundo Álvarez-Gavilanes;

Gonzalo Iván Andrade-Mena

\section{Oferta}

La oferta existente en la provincia del Azuay de este tipo de productos son distribuidores de partes y piezas para vehículos a diésel.

Tabla 7.

Porcentaje de participación de competencia en el mercado.

\begin{tabular}{ll}
\hline Competencia & $\begin{array}{l}\text { Porcentaje } \\
\text { participación }\end{array}$ \\
\hline Eco diésel & $23.10 \%$ \\
\hline Más diésel & $23.10 \%$ \\
\hline Diésel trucks & $19.20 \%$ \\
\hline Mavesa & $10.20 \%$ \\
\hline Otros & $24.40 \%$ \\
\hline Total & $100 \%$ \\
\hline
\end{tabular}




\section{CIENCIAMATRIA}

Revista Interdisciplinaria de Humanidades, Educación, Ciencia y Tecnología

Año VII. Vol. VII. N¹2. Enero - Junio. 2021

Hecho el depósito de ley: pp201602FA4721

ISSN-L: 2542-3029; ISSN: 2610-802X

Universidad Nacional Experimental Francisco de Miranda (UNEFM). Santa Ana de Coro. Venezuela

Francisco Agustín Zea-Izquierdo; Juan Edmundo Álvarez-Gavilanes;

Gonzalo Iván Andrade-Mena

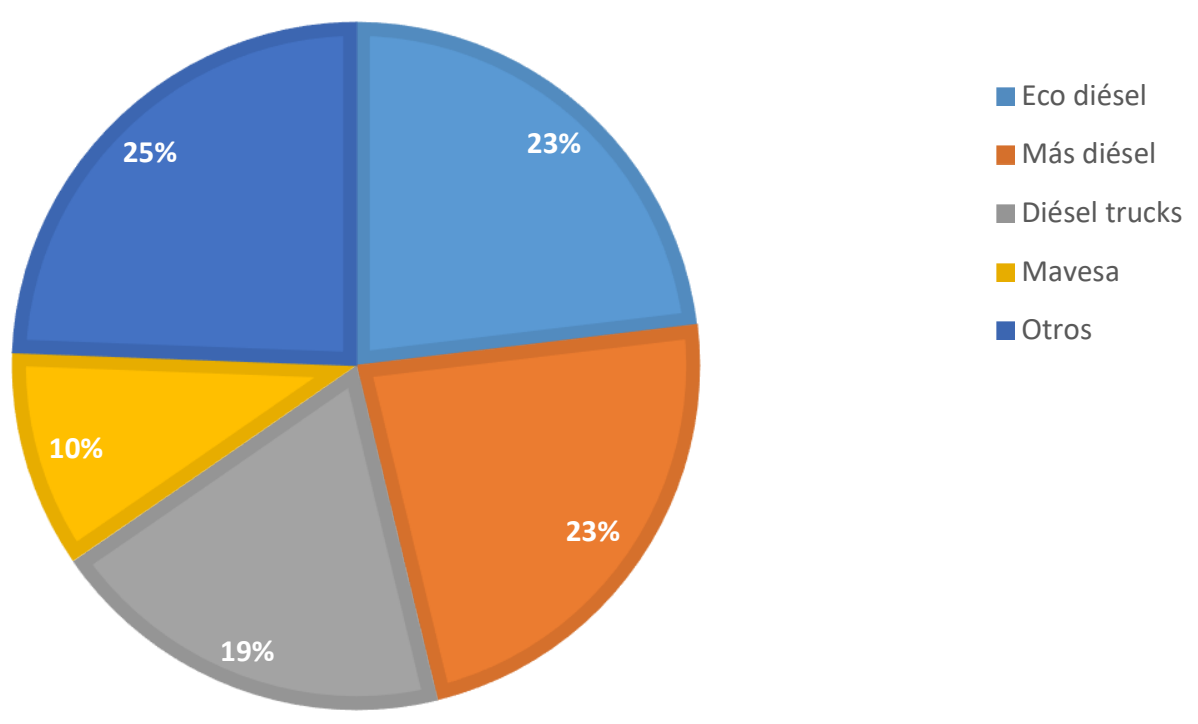

Figura 5. Porcentaje de participación de competidores.

Como podemos observar en el grafico anterior existen dos competidores que abarcan la mayor parte del mercado objetivo, mientras el resto del mercado se encuentra distribuido para otras empresas por lo que con una correcta planeación sería factible la entrada a este mercado. 


\section{CONCLUSIONES}

Como se ha podido observar a lo largo de este estudio, el análisis de mercado es una herramienta muy importante para analizar la factibilidad de la entrada de un inversor a un mercado determinado.

El estudio de mercado realizado nos indica que es factible invertir en este sector ya que está en constante desarrollo y crecimiento año a año.

Al poder conocer lo que el cliente espera del producto que se le va a ofrecer, la competencia a la que va a enfrentar y como hacer llegar los productos hasta el consumidor final el empresario logra tener un conocimiento más preciso y concreto del negocio al que entra y puede idear estrategias que le sirvan para posicionarse y luego mantenerse en el mercado.

Al realizar un análisis de mercado las empresas ahorran mucho tiempo y esfuerzo ya que al aprovechar los datos obtenidos se pueden saber las fortalezas y debilidades de la competencia y de los productos que estos ofrecen.

Se determinó además que los vehículos diésel en la provincia del Azuay representa una buena parte del total del mercado de vehículos y que existen muy pocos competidores dedicados a la distribución de partes y piezas de carros que utilizan este tipo de combustible.

\section{REFERENCIAS CONSULTADAS}

Álvarez, C., Narváez, C., Erazo, J., \& Luna, K. (2020). Lógica difusa como herramienta de evaluación del portafolio de inversiones en el sector cooperativo del Ecuador [Fuzzy logic as an investment portfolio evaluation tool in Ecuador's cooperative sector] Revista Espacios, 41(36). Obtenido de https://n9.cl/bai6c

Asociación de Empresas Automotrices Del Ecuador AEADE. (2018). Anuario 2018 [2018 Yearbook]. Asociación de Empresas Automotrices Del Ecuador AEADE, 40. Obtenido de https://n9.cl/w30p 
C.E.E.I Galicia, S.A. (BIC Galicia). (2010). Manuales prácticos de la pyme, cómo realizar un estudio de mercado [Practical manuals of sme, How to conduct a market study]. Santiago de Compostela: C.E.E.I Galicia, S.A. (BIC Galicia). Obtenido de https://n9.cl/q43ey

Carrillo, D. (2009). Diagnóstico del sector automotriz [Diagnostics of the automotive sector]. https://n9.cl/32yg

Coba, G. (2020). La venta de camiones crece en medio de la crisis de Covid-19 [Truck sales grow in the midsst of the Covid-19 crisis]. Primicias, p. 1. Obtenido de https://n9.cl/hq9p

Dvoskin, R. (2004). Fundamentos de marketing, teoría y experiencia [Marketing fundamentals, theory and experience]. Buenos Aires: Ediciones Granica. Obtenido de https://n9.cl/f80ir

Erazo, J. C., \& Narváez, C. I. (2020). La gestión del capital intelectual y su impacto en la efectividad organizacional de la industria de cuero y calzado en la Provincia de Tungurahua - Ecuador [The management of intellectual capital and its impact on the organizational effectiveness of the leather and footwear industry in the Province of Tungurahua - Ecuador]. Revista Espacios, 41(21), 254-271.

Fernández-Nogales, Á. (2004). Investigación y tecnicas de mercado. Madrid: ESIC. Obtenido de https://n9.cl/2if8

Ferré-Trenzano, J., \& Ferré-Nadal, J. (1997). Los estudios de mercado [The market studies]. Madrid: Ediciones Díaz de Santos S.A.

García-González, A., \& Bória-Reverter, S. (2006). Los nuevos emprendedores. Creación de empresas en el siglo XXI [The new entrepeneurs. Creation of companies in the 21st century]. Barcelona: Gráficas Rey S.L. Obtenido de https://n9.cl/vtnxq

Griffin, R., \& Ebert, R. (2005). Negocios [Business]. México: Pearson Educacion.

Instituto Latinoamericano de Planificación y Economía Social ILPES. (2006). Guía para la presentación de proyectos. [Guide for the presentation of projects]. México: Siglo XXI editores S.A de C.V. 
CIENCIAMATRIA

Revista Interdisciplinaria de Humanidades, Educación, Ciencia y Tecnología

Año VII. Vol. VII. N¹2. Enero - Junio. 2021

Hecho el depósito de ley: pp201602FA4721

ISSN-L: 2542-3029; ISSN: 2610-802X

Universidad Nacional Experimental Francisco de Miranda (UNEFM). Santa Ana de Coro. Venezuela

Francisco Agustín Zea-Izquierdo; Juan Edmundo Álvarez-Gavilanes;

Gonzalo Iván Andrade-Mena

Instituto Nacional de Estadística y Censo INEC. (2012). Análisis sectorial sector automotriz [Sectorial analysis of the automotive sector]. Infoeconomía, 1-2. Obtenido de https://n9.cl/nd673

Instituto Nacional de Estadística y Censos INEC. (2018). Ecuador en cifras, Tabulados del anuario de transporte [Ecuador in numbers,transport yearbook tabulations]. Obtenido de https://n9.cl/xwsrh

Instituto Nacional de Estadística y Censos INEC. (2019). Anuario de Estadísticas de transporte 2018 [Yearbook of statistics of transportation 2018]]. Quito: INEC. Obtenido de INEC: https://n9.cl/p6ev

Instituto Nacional de Estadísticas y Censos INEC. (2019). Boletín técnico anuario de estadísticas de transporte 2018 [Technical Boletin transport statistics yearbook 2018]. Quito: INEC. Obtenido de https://n9.cl/grg3

Kinnear, T., \& Taylor, J. (1991). Investigación de mercados un enfoque aplicado [Market research an applied approach]. Bogotá: McGraw Hill Latinoamericana. Obtenido de https://n9.cl/865lj

Kotler, P., \& Keller, K. (2006). Direccion de marketing [Marketing management]. México: Pearson Educación. Obtenido de https://n9.cl/1a7e

Luna-González, A. C. (2016). Plan estratégico de negocios. [Strategic business plan]. México: Grupo Editorial Patria, S.A de C.V.

Olea-Suaréz, D., \& Pacheco-Garcia, R. (2012). Marketing la administración y la gerencia básica para las mipymes colombianas Micros pequeñas y medianas empresas. [MArketing the administration and basic management for colombian micro, small and medium enterprises] (Primera ed.). Bogotá: Departamento de Publicaciones y comunicación gráfica de la Universidad Piloto de Colombia. Obtenido de https://n9.cl/vkjdb

Organización internacional del trabajo. (2005). Tendencias de la industria automotriz que afectan a los proveedores de componentes [Automotive industry trends affecting component suppliers]. Ginebra: Oficina Internacional del Trabajo OIT. Obtenido de https://n9.cl/tbej

Ortega-Bardellini, J. (2005). Análisis del sector automotor ecuatoriano [Analysis of the ecuadorian automotive sector]. Quito: Banco central del Ecuador. Obtenido de https://n9.cl/aosht 


\section{CIENCIAMATRIA}

Revista Interdisciplinaria de Humanidades, Educación, Ciencia y Tecnología

Año VII. Vol. VII. N¹2. Enero - Junio. 2021

Hecho el depósito de ley: pp201602FA4721

ISSN-L: 2542-3029; ISSN: 2610-802X

Universidad Nacional Experimental Francisco de Miranda (UNEFM). Santa Ana de Coro. Venezuela

Francisco Agustín Zea-Izquierdo; Juan Edmundo Álvarez-Gavilanes;

Gonzalo Iván Andrade-Mena

Parmerlee, D. (1999). Preparación del plan de marketing [Preparation of the marketing plan]. Barcelona: Granica. Obtenido de https://n9.cl/2w7ii

Pedraza-Rendon, O. H. (2014). Modelo del Plan de negocios para la micro y pequeña empresa [Model business plan for micro and small enterprises]. Mexico: Grupo Editorial Patria.

Sapag-Chain, N. (2007). Proyectos de inversión, formulación y Evaluación [Investment projects formulation and evaluation]. México, Mexico: Pearson educación de Mexico S.A.

Sellers-Rubio, R., \& Casado-Díaz, A. (2013). Introduccion al marketing Teoría y Practica [Introduction to marketing theory and practice]. Alicante, España: Club Universitario.

Sutil-Martín, L. (2013). Neurociencia, empresa y marketing [Neuroscience, business and marketing]. Madrid: ESIC. Obtenido de https://n9.cl/vbas|

Talaya, Á., Madariaga, J., Narros, M., Olarte, C., Reinares, E., \& Saco, M. (2008). Principios de marketing [Marketing Principles]. Madrid: ESIC. Obtenido de https://n9.cl/z48d

Vélez-Pareja, I. (2003). Decisiones empresariales bajo riesgo e incertidumbre [Business decisions under risk and uncertainty]. Bogotá: Grupo Editorial Norma.

Zhingre-Orellana, S., Erazo-Álvarez, J., Narváez-Zurita, C., \& Paulina Moreno, V. (2020). Plan de negocios para evaluar la viabilidad en el sector microempresarial de repuestos de vehículos. Revista Arbitrada Interdisciplinaria Koinonía, 5(10), 370396.

(C2021 por los autores. Este artículo es de acceso abierto y distribuido según los términos y condiciones de la licencia Creative Commons Atribución-NoComercial-Compartirlgual 4.0 Internacional (CC BY-NC-SA 4.0) (https://creativecommons.org/licenses/by-nc-sa/4.0/). 